\title{
PEAT: an intelligent and efficient paired-end sequencing adapter trimming algorithm
}

\author{
Yun-Lung Li' ${ }^{1}$, Jui-Cheng Weng ${ }^{1}$, Chiung-Chih Hsiao' ${ }^{1}$ Min-Te Chou' ${ }^{1}$, Chin-Wen Tseng ${ }^{1}$, Jui-Hung Hung ${ }^{1,2^{*}}$ \\ From The Thirteenth Asia Pacific Bioinformatics Conference (APBC 2015) \\ HsinChu, Taiwan. 21-23 January 2015
}

\begin{abstract}
Background: In modern paired-end sequencing protocols short DNA fragments lead to adapter-appended reads. Current paired-end adapter removal approaches trim adapter by scanning the fragment of adapter on the $3^{\prime}$ end of the reads, which are not competent in some applications.

Results: Here, we propose a fast and highly accurate adapter-trimming algorithm, PEAT, designed specifically for paired-end sequencing. PEAT requires no a priori adaptor sequence, which is convenient for large-scale metaanalyses. We assessed the performance of PEAT with many adapter trimmers in both simulated and real life pairedend sequencing libraries. The importance of adapter trimming was exemplified by the influence of the downstream analyses on RNA-seq, ChIP-seq and MNase-seq. Several useful guidelines of applying adapter trimmers with aligners were suggested.
\end{abstract}

Conclusions: PEAT can be easily included in the routine paired-end sequencing pipeline. The executable binaries and the standalone $\mathrm{C}++$ source code package of PEAT are freely available online.

\section{Background}

The paired-end sequencing technology, modified from the well-known single-end sequencing technology on next generation sequencing (NGS) platforms, plays increasingly important roles in genomics. By sequencing 5' ends of two strands of a DNA (or cDNA) fragment, it provides not just nucleic contents but also positional information of the fragment, therefore is a powerful resource to resolve the assembly in repetitive regions or structural variants. In addition, it is capable of obtaining interacting long-range DNA fragments [1], conveying information on both strands of the sequenced DNA, resolving exon junctions [2], and many other applications [3].

A typical Illumina's paired-end sequencing technology is performed as follows: double strands of DNA fragments are both ligated with adapters and barcodes (when multiplexing), and then the 5 ' ends of the double strands are attached to the flow cell surface followed by many

\footnotetext{
* Correspondence: jhhung@nctu.edu.tw

'Institute of Bioinformatics and Systems Biology, National Chiao Tung University, Hsin-Chu, Taiwan

Full list of author information is available at the end of the article
}

bridge amplification cycles to generate clusters for better nucleotide synthesis and fluorescence imaging. Both strands of each DNA fragment can serve as sequencing templates by regenerating the clusters, and consequently paired-end reads are produced (Figure 1A). According to the paired-end sequencing protocol of Illumina, users are allowed to choose the size-selected length of the DNA fragments (or inserts) from 200 to 500 base pairs (bp) with the sequencing quantity up to 200 millions of reads. Paired-end reads obtained are of a machine specific sequencing length, such as $36 \times 2,75 \times 2$, or $100 \times 2 \mathrm{bp}$.

For DNA fragments that are at least as long as the prespecified sequencing length (e.g., $100 \mathrm{bp}$ ), the sequencing process initiated from the 5 ' ends of both strands will be constrained and terminated before sequencing the adapters, so that only the 'real' DNA information is conveyed by the resultant paired-end reads (Figure 1A). However, in the case when the DNA fragments are shorter than $100 \mathrm{bp}$, the sequencer will 'read through' the real DNA into the adapters (Figure 1B). As a result, the paired-end reads generated will be appended with unwanted adapter 


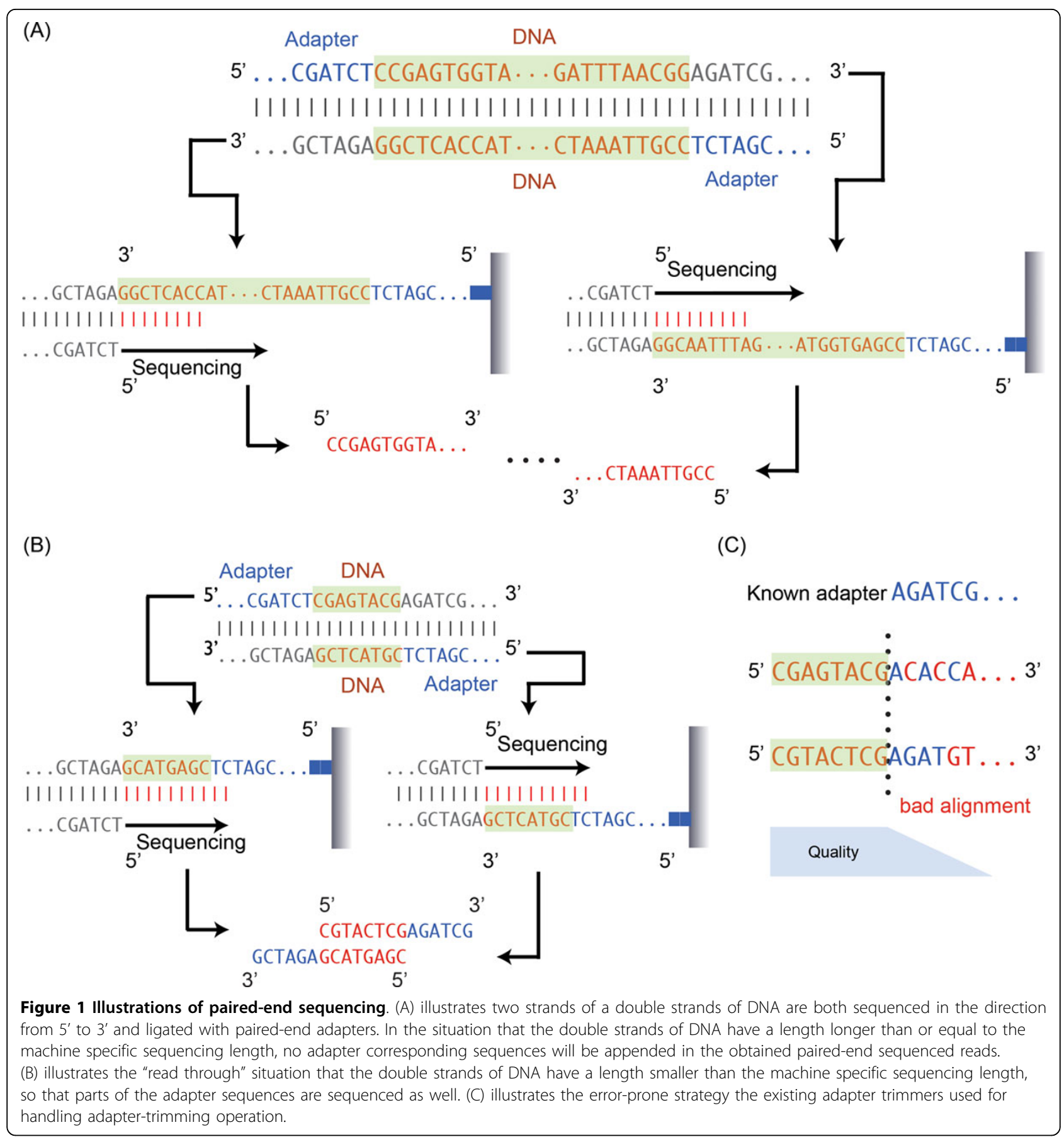

sequences (namely, adapter contamination), and are likely dropped in the step of reference mapping.

To recover the "real" DNA part from the reads, existing algorithms rely on the correct alignment between adapter sequences against the 3' ends of reads. Such algorithms were originally designed for dealing with adapter contamination in single-end sequencing. They regard paired-end reads as two sets of single-end reads, and the adapter trimming is performed on each set independently. In practice, these single-end oriented adapter trimmers, such as FASTX [4], Cutadapt [5], ea-utils [6], TagCleaner [7], and Trim_Galore [8] (a wrapper of cutadapter for paired-end sequencing), do not give satisfactory results for paired-end sequencing. It is largely because current modern sequencers have a significantly higher sequencing error rate at the 3 ' ends of reads (Figure 1C). In other words, identifying the putative trimming positions by means of matching adapter sequence against read segments with poor sequencing 
qualities is highly error-prone. Some trimmers, such as AdapterRemoval [9], SeqPrep [10], and Trimmomatic [11], further filter positions found by the adapter sequencing scanning on each of the paired reads by examining the reversed complementarity between the untrimmed portions of the paired reads; however the accuracy still depends on the adapter sequence scanning on the error prone 3' ends. GATK ReadAdaptorTrimmer [12] looks for overlaps between forward and reverse reads in a pair to achieve high accuracy; however, it was designed specifically for streamlining with variant calling analysis pipeline in GATK [13], its input and output has to be in SAM/BAM format [14], which is cumbersome for other downstream analysis. The comparison of the performance of some of the trimmers has been investigated by several benchmark studies [15-17].

Here, we propose a highly efficient and accurate adapter-trimming algorithm and its implementation, PEAT (Paired-End Adapter Trimmer), designed specifically for paired-end sequencing. PEAT requires no adapter sequence input, which is particularly convenient when processing libraries using different adapters on a large scale. PEAT directly scans for the reverse complementarity between the good quality portions of the reads to avoid the loss of sensitivity during the filtering adopted by conventional approaches. We compared PEAT with many adapter-trimming tools. PEAT performed relatively well in simulated benchmarks and showed high scalability when applied to large real datasets. We applied PEAT to two public real datasets $(101 \times 2$ paired-end sequencing libraries with 150 millions of total sequencing reads), multimillions of adapter-appended reads were successfully spotted, recovered, and mapped back to the reference. We further investigated the effects of PEAT to ten more real publicly available datasets of different sequencing applications such as ChIP-seq, MNase-seq, and RNA-seq. Comparisons between datasets processed with and without PEAT followed by the same typical downstream analyses revealed obvious pattern changes, which may deflect the biological notions toward the data. We suggest that more attentions need to be paid to adapter contamination in analyzing paired-end reads in all applications.

\section{Results and discussion}

\section{An overview of the PEAT algorithm}

In a typical Illumina paired-end sequencing protocol, a pair of adapter-appended reads emerges whenever a DNA fragment shorter than the pre-specified sequencing length get sequenced (Figure 1B). For a pair of reads sequenced from such a short fragment, the 5 ' ends of the reads, which correspond to the sequences of the real DNA fragment, have to be the reverse complement to each other (barcoding is ignored for simplification). The
3 ' ends, on the other hand, are corresponding to the adapter sequences, and should be equally long due to the fix pre-specified sequencing length.

Based on the aforementioned observations, we propose a paired-end adapter-trimming (PEAT) algorithm with an efficient two-stage string matching strategy to detect the junctions between real DNA and adapters in paired-end libraries (Figure 2). The algorithm identifies multiple possible trimming positions by probing the reverse complementarity of the $5^{\prime}$ end of the paired reads. To begin with, the reverse complement of a short $5^{\prime}$ prefix ( $L$ nucleotides in length) of one read of the pair is used as a template to scan for mismatch-tolerant hits against another read of the pair. This procedure is performed twice by taking each read of a pair as the template iteratively and generates two possible sets of putative trimming sites. The intersection of the two sets is then used to check the reverse complementarity of the accordingly determined real DNA sequences (based on the given putative trimmed sites) of each read in a pair, and the parts corresponding to the adapter sequences, are substantially the same (in the case that forward strand and reverse strand adapters used in sequencing library preparation are totally different, the latter step can be skipped, see Methods). If none of the trimming position passes the check, PEAT reports that the pair is not appended with any adapter sequence.

By using 5' ends instead of 3' ends as both templates and targets for scanning, PEAT is able to determine the adapter trimming positions of the paired-end reads more accurately. PEAT is therefore more resistant to adapter contamination that is very short or having multiple adapter copies. Optionally, PEAT can also take the parts corresponding to error-prone adapter sequences as an auxiliary criterion for reexamination when the forward- and reverse-strand adapters share similar sequence (the $E_{3}$ parameter, see Methods). PEAT also incorporates an open source single-end adapter trimming algorithm [18] to provide single-end adapter trimming functionality. PEAT supports multithreading for utilizing multiple computing nodes with data parallelism. Detailed algorithms and implementation can be found in Methods and the source code.

\section{Performance comparison}

We evaluated the performance of PEAT, as well as other paired-end adapter trimmers, including AdapterRemoval, ea-utils, GATK ReadAdaptorTrimmer, SeqPrep, Trimmomatic, and Trim_Galore with six benchmark simulations and two real life datasets. The parameters of running these tools can be found in Supplementary Methods (Additional file 2). The benchmark simulations included datasets of three levels of quality: low-error-rate, middle-error-rate, and high-error-rate datasets, namely LED, MED and HED 


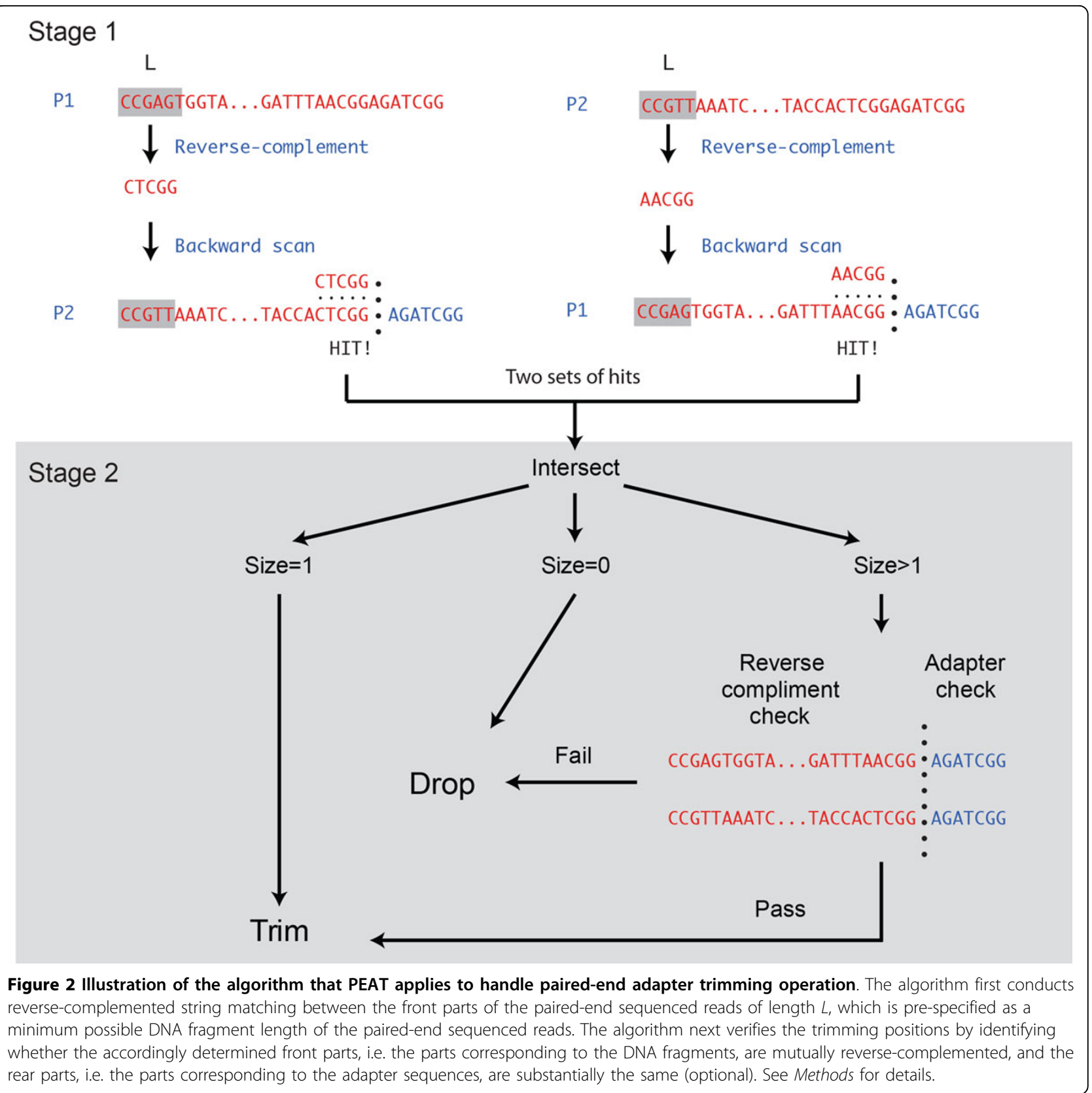

respectively, each of which had a million mock paired-end reads randomly sampled from a reference mouse genome (mm9) and half of the paired-end reads were selectively appended with the typical Illumina adapter sequences. Since typical Illumina libraries use the similar forwardand reverse-strand adapters, we also generated three companion dLED, dMED and AHED of which the prefix ' $d$ ' indicated that the reads were generated with the distinct forward- and reverse-strand adapters. In addition, we generated MED-o/dMED-o in which each paired-end read shared a 50 -bp overlap (designated by the suffix -o) to demonstrate that our algorithm would not be fooled by extra reverse complementarity within reads. Mutations were introduced into reads according to a set of given sequencing quality scores (Figure 1, Additional file 1). The quality scores were sampled from a real dataset. Please see Methods for more details.

We ran PEAT and other trimmers on a Linux virtual machine and measure the execution time for trimming $1 \mathrm{M}$ of simulated reads of each of the eight datasets. All in all, with a linear-time algorithm (running time is proportional to the number of total bases, see Methods) 
effectively implemented with a lower-level computer language (i.e., $\mathrm{C}++$ ), PEAT clearly achieved higher efficiency (FIG. S2, Additional file 1).

To evaluate the trimming performance, we calculated the sensitivity, specificity, accuracy, and Matthews correlation coefficient (MCC) [19] of all trimmers (FIG. S3, Additional file 1). Since some trimmers trim bases with low quality at 3' end and throw away reads that are too short after trimming by default, we used the options provided by the tools to make them behave similarly to the scenarios (see Supplementary Methods, Additional file 2). PEAT topped at two general metrics (accuracy and $\mathrm{MCC}$ ) in most of the datasets. In general, the accuracy deteriorated when different adapters were used. AdapterRemoval seemed intolerant of different adapters in the paired-end libraries. Overlapping reads did not make apparent difference to the performance of all trimmers. We further investigated the ratio of trimmed reads over untrimmed reads (expected ratio: 1; Figure $3 \mathrm{~A}$ and S4-6A, Additional file 1) and the length distribution of



(B)



(C)

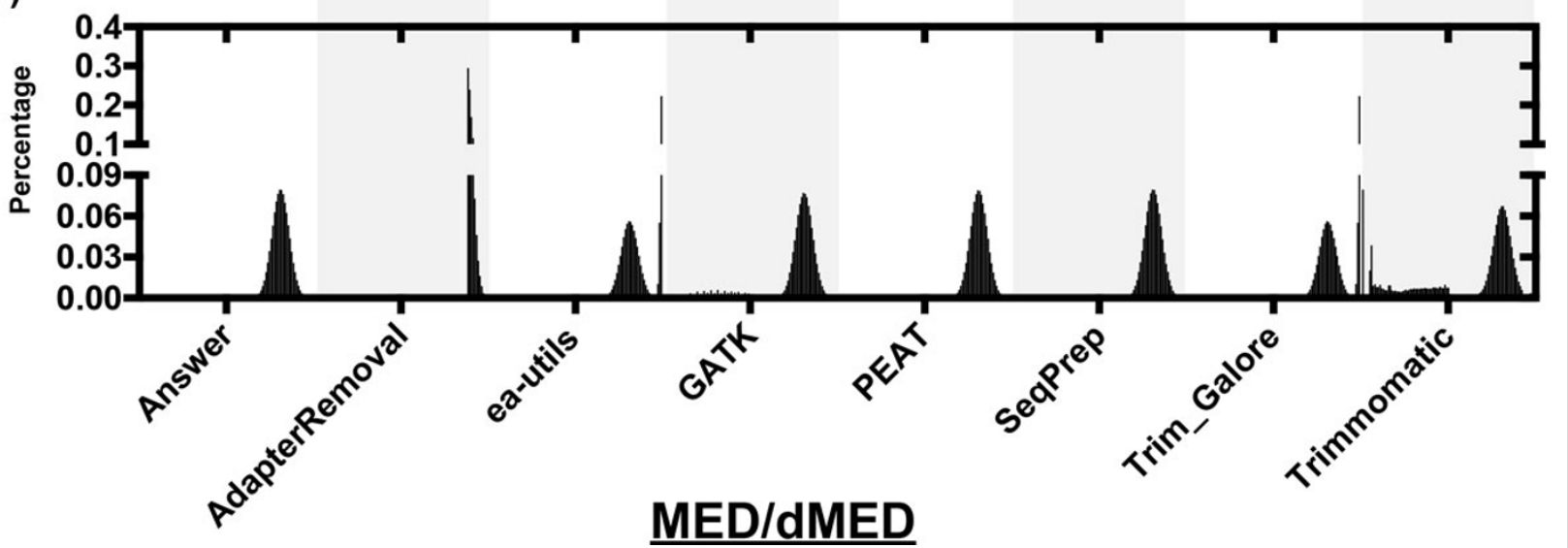

Figure 3 Middle-error-rate (MED) datasets. (A) shows the ratio of the read count of the untrimmed reads over that of the trimmed reads by all tested trimmers applied to the simulated MED/dMED datasets. (B, C) illustrates the length distributions of the trimmed reads processed by each of the tested adapter trimmers applied to simulated MED (B) and AMED (C) datasets. The distributions are depicted with the ratio of the amount of reads trimmed at certain length, ranging from 1 to $100 \mathrm{bp}$, over the total amount of trimmed reads. The ratios are magnified by 5 times with the range from 1 to 50 to visualize the presence of short fragments after trimming. 
trimmed reads (Figure $3 \mathrm{BC}$ and $\mathrm{S} 4-6 \mathrm{BC}$, Additional file 1) obtained in eight datasets. The length distribution shows that some trimmers tended to trim a few bases at the 3' end (e.g., ea-utils and Trim_Galore), and some resulted in short fragments with a small chance (e.g., Trimmomatic and GATK ReadAdaptorTrimmer). Overall, the results of GATK ReadAdaptorTrimmer, PEAT, and SeqPrep showed only marginal differences to the gold standards.

We next applied PEAT and other trimmers to real life paired-end datasets $(101 \times 2)$ retrieved from GEO (GSM929772 and GSM929773, see Methods). In the comparison of trimming time with other tools, PEAT operated faster than AdapterRemoval, ea-utils, Trim_Galore, GATK ReadAdaptorTrimmer, SeqPrep and Trimmomatic by remarkable speedups of 20X, 2.4X, 2.7X, 9X, 13X, 7X respectively (see Table 1; no parallelism was applied in all the tests.). The result consists with that in the benchmark simulations, suggesting that PEAT is more efficient and also more scalable than most of the existing trimmers.

PEAT identified 7.71M (5.04\%) and 10.59M (5.05\%) adapter-appended reads from GSM929772 and GSM929773 respectively (Table 1). The identified adapterappended reads were further aligned back to the $\mathrm{mm} 9$ reference with end-to-end alignment by Bowtie2 [20]. $5.99 \mathrm{M}$ and $8.79 \mathrm{M}$ reads were concordantly aligned one or more times to the genome, indicating that PEAT successfully had $77.68 \%$ and $83.03 \%$ of the identified adapterappended reads of the two datasets properly processed and recovered. Since there was no gold standard, we simply took the number of reverse-complemented trimmed pairs to approximate true positives, and denoted it as aTP. PEAT gave the highest aTP among all tools. Comparing the insert length distribution of the original datasets to that processed by PEAT (FIG. S7 and S8, Additional file 1), we conclude that PEAT successfully recovered a significant amount of inserts shorter than $101 \mathrm{bp}$ without losing reads information conveyed in the original datasets.
For our tests, the results suggest that although some other trimmers also take advantage the reversecomplementarity to identify adapter contamination in different ways and also showed comparably performance, their algorithms do not scale very well and took much more time to complete the task. In addition, GATK ReadAdaptorTrimmer required extra 20+ hours of time just to transform between the formats for the two real life datasets (GSM929772 and GSM929772) in the tests. It could be a burden for users that do not intent to use the GATK framework.

\section{Applications in RNA-seq, ChIP-seq, and MNase-seq}

In practice, the importance of adapter trimming is not fully recognized. It is not rare to assume that after sizeselection-a common procedure to eliminate unwanted short fragments such as degradation products or rRNAsDNA fragments having length shorter or greater than the size-selected length by a margin take only a very tiny part of the sequencing library. According to this assumption, DNA fragments with lengths outside the peak (either shorter or longer) are extremely rare, which makes some believe the lost of information carried by these minority is tolerable. We applied PEAT to two real life RNA-seq datasets GSM929772 and GSM929773 and provided evidence that this assumption might need to be adjusted (see FIG. S9, Additional file 1 and Supplementary Methods, Additional file 2).

We further collected publicly available pairedend sequencing datasets for three typical NGS applications from GEO: RNA-seq (GSM929772-3, GSM1000574_1, GSM1000574_3), ChIP-seq (GSM862560_r1, GSM862561_r1, GSM862562_r1, GSM862563_r1) and MNaseseq (GSE58101_1-3), and we tested the influence on the downstream analyses when a competent adapter trimmer (i.e., PEAT) is included in the data processing. We used the local alignment option (-local) in Bowtie2 to relieve the problem of the non-mappability of the adapter-appended

Table 1 Performance comparison of tested trimmers applied to two real life datasets

\begin{tabular}{|c|c|c|c|c|c|c|c|}
\hline & PEAT & $A R^{*}$ & $\mathrm{EA}^{*}$ & $\mathrm{TG}^{*}$ & GATK $^{*}$ & SeqPrep & $\mathrm{TM}^{*}$ \\
\hline \multicolumn{8}{|c|}{ GSM929772 (sequence depth: 152.8M) } \\
\hline Time & $1 \mathrm{~h} 40 \mathrm{~m}$ & $36 \mathrm{~h} 35 \mathrm{~m}$ & $3 \mathrm{~h} 59 \mathrm{~m}$ & $4 \mathrm{~h} 31 \mathrm{~m}$ & $14 \mathrm{~h} 51 \mathrm{~m}$ & $22 \mathrm{~h} 49 \mathrm{~m}$ & $12 \mathrm{~h} 07 \mathrm{~m}$ \\
\hline \# Trimmed & $7,705,821$ & $2,568,156$ & $5,444,126$ & $15,023,863$ & $6,843,371$ & $5,795,255$ & $15,298,851$ \\
\hline \# Mappable & $5,985,640$ & $2,107,352$ & $4,735,584$ & $11,160,564$ & $5,562,822$ & $5,031,866$ & $11,158,090$ \\
\hline aTP & $7,088,576$ & $2,507,720$ & $5,426,673$ & $4,682,889$ & $6,444,882$ & $5,789,997$ & $3,032,621$ \\
\hline \multicolumn{8}{|c|}{ GSM929773 (sequence depth: 209.7M) } \\
\hline Time & $1 \mathrm{~h} 32 \mathrm{~m}$ & $33 \mathrm{~h} 30 \mathrm{~m}$ & $3 \mathrm{~h} 40 \mathrm{~m}$ & $3 \mathrm{~h} 54 \mathrm{~m}$ & $13 \mathrm{~h} 55 \mathrm{~m}$ & $20 \mathrm{~h} 07 \mathrm{~m}$ & $10 \mathrm{~h} 51 \mathrm{~m}$ \\
\hline \# Trimmed & $10,587,748$ & $3,243,421$ & $8,659,761$ & $17,427,938$ & $10,191,334$ & $9,070,298$ & $22,184,570$ \\
\hline \# Mappable & $8,791,108$ & $2,708,858$ & $7,604,812$ & $13,991,491$ & $8,755,726$ & $7,981,609$ & $17,451,029$ \\
\hline aTP & $10,317,323$ & $3,177,176$ & $8,535,723$ & $7,535,502$ & $10,063,241$ & $9,062,206$ & $5,046,115$ \\
\hline
\end{tabular}

"Abbreviations: AR(AdapterRemovel); EA(ea-utils); TG(Trim_Galore); GATK (GATK ReadAdaptorTrimmer); TM(Trimmomatic) 
reads, which is a generally acceptable approach for the purpose [21] (see Supplementary Methods, Additional file 2). To eliminate possible miscalculation of insert length, we further excluded the multi-mapping reads and found significant changes in the length distribution (Unique mapping reads only: Figure 4 and S10, Additional file 1; with multimapping reads: FIG. S11 and S12, Additional file 1).

In ChIP-seq datasets, we found that, without applying PEAT, unique mapping reads were reduced for about 40\% (Figure 4A and S10A, Additional file 1), but after

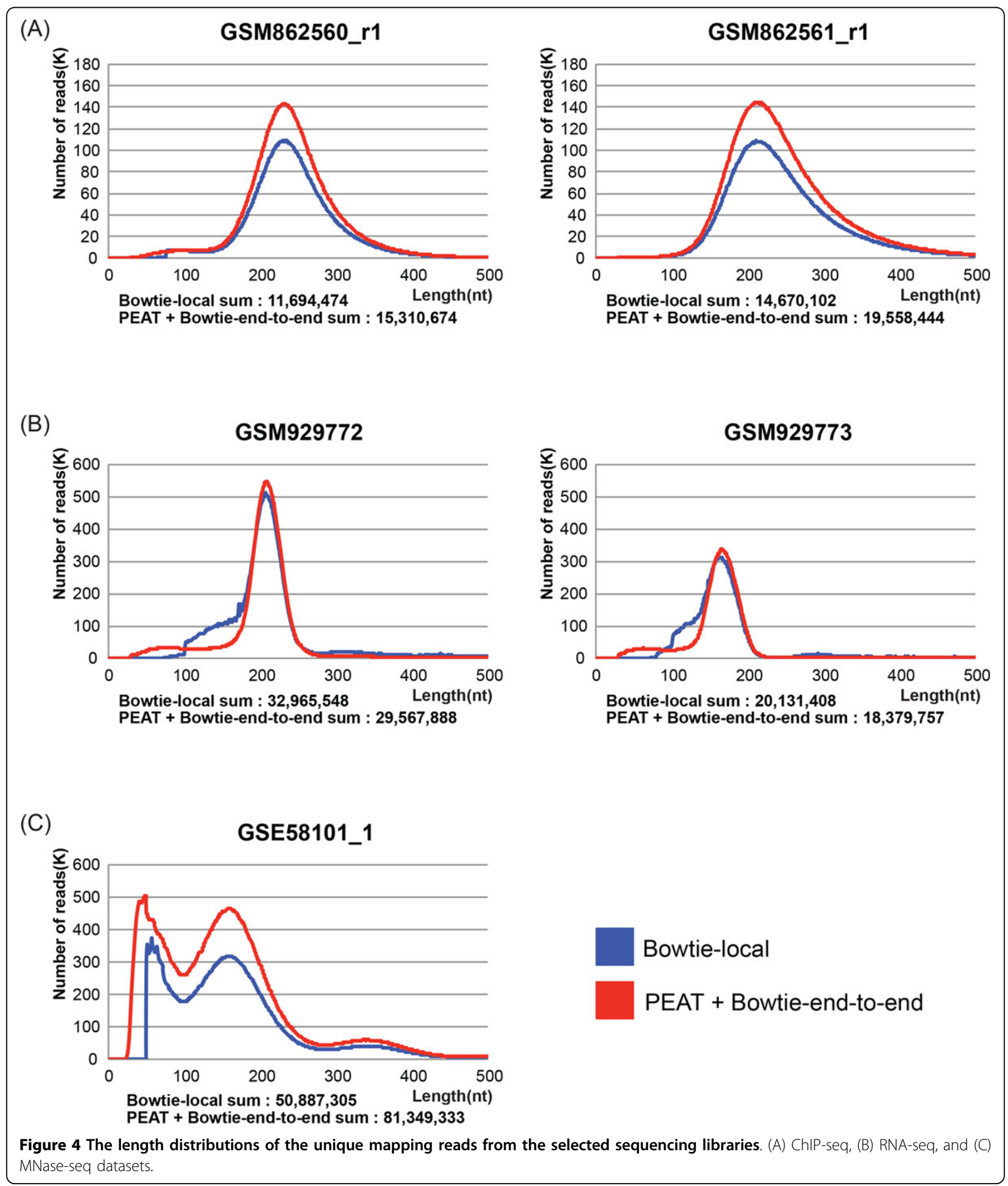


closer examination, the reduction were largely due to the use of local alignment, which led to fewer unique alignment. We didn't observe significant influence to the power of the downstream peak calling with adapter trimming (FIG. S13, Additional file 1. KS-test p-value: 0.051, 0.08, 0.068, and 0.052 for GSM862560_r1, GSM862561_r1, GSM862562_r1, GSM862563_r1 respectively); however, we would still suggest one should perform adapter trimming with Bowtie end-to-end alignment, since loosing $40 \%$ of unique mapping reads might be too costly just for getting rid of adapter contamination. In RNA-seq datasets, contrary to that in ChIP-seq and MNase-seq datasets (see below), about $10 \%$ more unique mapping reads were found without PEAT. We can see an additional surge of the blue curve (without PEAT) around 100-200 bp in Figure 4B and S10B, Additional file 1. We examined some of these alignments and found they were likely caused by the truncated junction reads (see FIG. S14, Additional file 1 for a typical case); therefore likely all the junction reads were reported shorter. However, using Bowtie2 with the end-to-end alignment option threw away all these junction reads. The best practice here should be using PEAT followed by a splice junction mapper like TopHat [22]. The short (<100 bp) and long (>100 bp) inserts found with PEAT were annotated to different populations (see Supplementary Methods, Additional file 2). Short inserts were enriched in snoRNAs and they were not found in the results without PEAT. In MNase-seq datasets, more than $30 \%$ of unique mapping reads were missed without PEAT (Figure 4C and 10C, Additional file 1). The absence of the short inserts affected the downstream V-plot analysis. The CTCF occupation profiles were less visible without adapter trimming, which may lead to wrong estimation of the size of the binding motifs (see Methods, Figure 5 and FIG. S15, Additional file 1). Along with these observations, we strongly suggest that a competent adapter trimming is crucial for the correct interpretation of the paired-end sequencing data.

\section{Conclusions}

We herein propose the first paired-end specific adapter trimming algorithm and its implementation, namely PEAT. PEAT acknowledges the characteristics of current paired-end sequencing and takes advantage of the self reverse-complementarity nature of adapter-appended read pairs. It exploits a two-step process to search for reverse complement portions and accommodate high sequencing errors at the 3' ends. PEAT is written in C++ for more sophisticate control over memory and also equipped with multithreading for multiprocessor systems. Besides, with its reverse complementarity examining algorithm for 5' end, PEAT is able to operate without any adapter sequence input. This feature makes PEAT more suitable than other tools to handle large-scale sequencing data processes, in which different adapter sequences were used during library preparation.

Our simulation showed that single-end oriented trimmers that utilize post-processing to handle paired-end


Figure 5 The V-plots of the selected MNase-seq datasets. The anchors were CTCF binding sites. The plot in the left panel was processed with PEAT and Bowtie2 end-to-end alignment option; the plot in the right panel was processed with Bowtie2 local alignment option. 
reads might be inappropriate. PEAT was able to determine the adapter trimming position of the paired-end sequenced reads with impressively high accuracy and efficacy. For the tests on real life datasets without gold standards, we used aTP to estimate the performance of trimmers and showed that PEAT was among the best choices. In addition, typical downstream analyses of ChIP-seq, RNA-seq, and MNase-seq were carried out and the results underlined the importance of a competent adapter trimmer.

PEAT currently is unable to handle barcode sequences in 5' ends, which will be improved in the future development. PEAT does not take into account of quality scores and indels. It is possible for PEAT to adopt a probabilistic model for scanning hits, although it is unpredictable if the model would help for better sensitivity or specificity, but it would likely reduce the speed. For sequencing platforms that introduce indels into reads (such as the 454 sequencers), PEAT doesn't perform well, but in the algorithmic aspect, it is not difficult to allow a fix number of gaps when scanning for hits without lose of efficacy. In addition, SOLiD reads in color space can be converted into nucleotide before processing by PEAT. We will support gapped alignment and color space alignment in the near future. The executable binaries and the standalone $\mathrm{C}++$ source code package of PEAT are available at: [http://jhhung.github.io/PEAT].

\section{Methods}

The rationale behind PEAT is to find the mutually reversecomplement portion between two paired reads. A naive implementation for an exhaustively search of all possible trimming sites of one read pair takes quadratic time. PEAT employs a two-stage algorithm to find the proper trimming positions in linear time without loss of specificity and sensitivity (Figure 1). The implementation details for the two stages of string matching are disclosed in the following paragraphs (see FIG. S16, Additional file 1).

\section{First stage of string matching}

In the first stage of string matching, PEAT starts with an initial string matching length $L$, which can be set according to the minimum size of DNA fragments of interest. In current implementation, $L$ is set as 30 by default. Let $R_{1}$ and $R_{2}$ denote the two reads of a pair. The first $L$ characters of $R_{1}$ and $R_{2}$, denoted by $T_{1}$ and $T_{2}$ respectively, are selected. $T_{1}$ is then reverse complemented and matched against $R_{2}$ with a pre-defined mismatch-tolerance level (see below). All possible occurrences of reverse-complemented $T_{1}$ in $R_{2}$ (denoted by $G_{1}$ ) signifies potential trimming sites where the 5 ' portion corresponding to the real DNA. PEAT performs the same matching procedure in the second round by aligning reverse-complemented $T_{2}$ against $R_{1}$, and obtaining another group of potential trimming sites $G_{2}$. PEAT then performs set intersection on $G_{1}$ and $G_{2}$, to further extract highly reliable trimming sites (denoted by $I$ ) that are reported twice. If $I$ is empty, PEAT reports $R_{1}$ and $R_{2}$ non-adapter-appended and works on the next read pair. If only one element is in I, PEAT simply reports it as the trimming site, and skips to the next read pair. While the intersection $I$ has at least two elements, PEAT proceeds to the second stage of string matching (see below). The total required comparison in matching step is $2^{*} L^{*} N$, where $N$ is the length of a read. Since $L$ is a small constant, the time complexity is therefore $\mathrm{O}(N)$.

\section{Second stage of string matching}

The biggest element $\left(I_{k}\right)$ in $I$ is taken, and the real DNA portions as well as adapter portions of $R_{1}$ and $R_{2}$ are retrieve according to $I_{k}$. PEAT further examines the validity of the current trimming position $\left(I_{k}\right)$ by determining whether the two real DNA portions are reversecomplement to their counterparts by an error tolerant string matching. PEAT also performs an optional operation to test if the two resultant adapter parts are similar in sequence (this operation is turned on by default, and can be turned off by setting $E_{3}=1$, see below) for libraries that use distinct 3 ' and 5' adapters). The validation of $I_{k}$ has $\mathrm{O}(N)$ time complexity. If the aforementioned criteria are met, PEAT accordingly regards the trimming position as valid, reports $I_{k}$, and proceeds to the next read pair, otherwise PEAT removes $I_{k}$ from $I$, and repeats the second stage of string matching until the intersection group $I$ becomes empty. Since $G_{1}$ and $G_{2}$ are constructed by scanning from the 5 ' end, they are sorted instinctually, $I$ can be obtained in $\mathrm{O}(|I|)$. The size of $I$ is small due to the small odds of having false positives (the sequencing errors more likely lead to false negatives than false positive), the cost can be amortized and the total time complexity of this stage is $\mathrm{O}(N)$.

\section{Error tolerant string matching}

PEAT achieves error tolerance by setting a threshold on the ratio of perfect matches between two strings. The threshold corresponding to the first stage of string matching $\left(T_{1}\right.$ v.s $R_{2}$ and $T_{2}$ v.s. $\left.R_{1}\right)$ is denoted by $E_{1}$, which indicates that the maximum mismatches allowed is $L^{*} \mathrm{E}_{1} . L$ and $\mathrm{E}_{1}$ should be carefully chosen for adequate error tolerance. There are two additional thresholds in the second stage: the ratios for the reverse complementarity check $\left(E_{2}\right)$ of the DNA parts and that for the equality check $\left(E_{3}\right)$ of the adapter parts of $R_{1}$ and $R_{2}$. To ease the burden picking parameters, we fully test the combination of all $E$ in different setups, and suggest to set $L=30, E_{1}=0.4, E_{2}=0.6$, and $E_{3}=0.4$. All three thresholds can be adjusted by users for better performance regarding different library preparations. Users 
can set thresholds to one to turn off the corresponding steps.

\section{Simulation dataset generation}

The system flow of the simulation dataset generation is illustrated in FIG. S17, Additional file 1. For generating adapter-appended read pairs, the length of each read was predetermined (101 nt), while the length of the adapter sequence (denoted as $A$ ) to be appended was sampled from a Gaussian distribution, $N(\mu=20, \sigma=5)$. Next, a reference genome, $\mathrm{mm} 9$, was used as a reference for generating a pair of adapter-appended reads. One randomly selected local sequence, with the length of the insert (i.e., 101-A), of the reference genome was obtained, and further reverse complemented to serve as its pairing counterpart. The well-conceived Illumina adapter sequences, 5'-AGATCGGAAGAGCGGTTCAGCAGGAATGCCGAGACCGATCTCGTATGCCGTCT TCTGCTTG (forward strand) and 5'-AGATCGGAAGAGCGTCGTGTAGGGAAAGAGTGTAGATCTCG GTGGTCGCCGTATCATT (reverse strand), were cropped and appended to the previously generated pair of reverse complemented sequence of reads to reach the predefined length. In the dHED, dMED, and dLED datasets, the adapter sequences used were 5'-CTAGAGTCAGTCCGGTTAATCCGGATCAGTCGTAGGAATCCAAAAGGTCCGTACGTACCTT (forward strand) and 5'-ATGGGCCCCTTTTAGTCAGTCAGTGGTTGGC CCTTTAAAATTTTCTCTTGAAGTCCCC (reverse strand). For adapter-free reads, on the other hand, two local sequences were randomly retrieved from the genome. In all cases, regions that have undetermined nucleotide were avoided. Each read was associated with quality scores based on the FastQ files acquired form a real dataset (GSM929772).

The encoded quality values were parsed and converted back to its numerical scales, each of which indicated the error probability of the corresponding nucleotide. In the generation of the MED, the error probabilities corresponding to each nucleotides were directly employed for mutation application, during the generation of the LED and HED, the error probabilities were scaled down or up by $10^{0.5}$ times respectively. Mutations were introduced to each position according to the error probability. After that, reads generated were output in FastQ format with a sequentially generated read index number as the identifier and the corresponding quality values. In addition, we kept track of the identifiers together with its corresponding actual trimming sites, numbers of mutations in both DNA and adapter portions for the follow-up evaluation. Consequently, three sets of one millions of simulated paired-end sequencing reads (half adapter-appended), each of which was constructed from a reference genome segment, were selectively appended with a randomly determined length of adapter sequences, and randomly applied sequencing errors were introduced.

\section{Real life datasets}

The RNA-seq datasets GSM929772, GSM929773, GSM1000574_1, GSM1000574_3 are all RNA-Seq libraries taken from Illumina Genome Analyzer provided by UCSC ENCODE data coordinating center. According to the documentation, polyA-selected RNAs were fragmented and converted into cDNA, and pairedend $2 \times 101 \mathrm{bp}$ reads were obtained from each end of a cDNA fragment. Paired-end libraries were further size-selected around $200 \mathrm{bp}$. The ChIP-seq datasets GSM862560_r1, GSM862561_r1, GSM862562_r1, GSM862563_r1 in was provided by GEO. The datasets were designed to determine the DNA binding sites of CTCF in mouse brain and contained paired $2 \times 76$ bp reads. Three MNase-seq datasets from GSE58101, which were called GSE58101_1, GSE58101_2 and GSE58101_3 in this paper.

\section{Downstream analyses \\ $\checkmark$ plot analysis}

The anchor CTCF binding sites were collected from Chen et al. [23]. We selected the top 1000 most significant peaks called by MACS 1.4 [24] with default setting from MNase-seq datasets and refined their borders by extending 20bp both up- and down-stream of the peak summits, which are the points with highest read coverage within peaks. We then generated V plots based on Henikoff et al. [25]. Transcriptome annotation: The annotations were gathered from the mouse genie informatics (MGI) [26]. Peak calling. The alignment results of ChIP-seq and corresponding input datasets were processed by MACS 1.4 with default setting.

\section{Additional material}

Additional file 1: Supplementary figures and legends

Additional file 2: Supplementary methods.

List of abbreviations

Next generation sequencing (NGS); Base pairs (bp); Low-error-rate dataset (LED); Middle-error-rate dataset (MED); and High-error-rate dataset (HED); Approximate true positives (aTP); Matthews correlation coefficient (MCC);

Competing interests

The authors declare that they have no competing interests.

\section{Authors' contributions}

$\mathrm{JHH}$ devised the algorithm and prepared the manuscript. YLL, JCW and JHH wrote the software. All authors contributed to the tests of the algorithm, presenting the results and preparing the figures. 


\section{Acknowledgements}

We thank the members of the Hung laboratories for helpful discussion and critical testing. Special thanks to anonymous reviewers, who gave valuable suggestions to the manuscripts.

\section{Declarations}

This work was supported in part by grants from National Science Council (102-2311-B-009-001 and 103-2221-E-009-128-MY2), National Chiao Tung University and Ministry of Education (Junior Faculty Research Enhancement Project and Aiming for the Top University Program) of Taiwan to $\mathrm{JHH}$. This article has been published as part of BMC Bioinformatics Volume 16 Supplement 1, 2015: Selected articles from the Thirteenth Asia Pacific Bioinformatics Conference (APBC 2015): Bioinformatics. The full contents of the supplement are available online at http://www.biomedcentral.com/ bmcbioinformatics/supplements/16/S1

\section{Authors' details}

${ }^{1}$ Institute of Bioinformatics and Systems Biology, National Chiao Tung University, Hsin-Chu, Taiwan. ${ }^{2}$ Department of Biological Science and Technology, National Chiao Tung University, Hsin-Chu, Taiwan.

Published: 21 January 2015

\section{References}

1. Fullwood MJaR Y: ChIP-based methods for the identification of longrange chromatin interactions. J Cell Biochem 2009, 107(1):30-39.

2. Le Hir $\mathrm{H}$ : The spliceosome deposits multiple proteins 20-24 nucleotides upstream of mRNA exon-exon junctions. The EMBO Journal 2000, 19(24):6860-6869.

3. Paired-End Sequencing | Achieve maximum coverage across the genome. [http://illumina.com].

4. FASTX-Toolkit. [http://hannonlab.cshl.edu]

5. Martin M: Cutadapt removes adapter sequences from high-throughput sequencing reads. EMBnetjournal 2011, 17(1):10-22.

6. Aronesty E: Comparison of Sequencing Utility Programs. The Open Bioinformatics Journal 2013, 7:1-8.

7. Schmieder R, Lim Y, Rohwer F, Edwards R: TagCleaner: Identification and removal of tag sequences from genomic and metagenomic datasets. BMC Bioinformatics 2010, 11:341.

8. Babraham Bioinformatics - Trim Galore! Babraham Bioinformatics - Trim Galore!. [http://www.bioinformatics.babraham.ac.uk/projects/trim_galore/].

9. Lindgreen S: AdapterRemoval: easy cleaning of next-generation sequencing reads. BMC Research Notes 2012.

10. SeqPrep. [https://github.com/jstjohn/SeqPrep]

11. Bolger AM LM, Usadel B: Trimmomatic: A flexible trimmer for Illumina Sequence Data. Bioinformatics 2014.

12. GATK. [http://www.broadinstitute.org/gatk].

13. McKenna A, Hanna M, Banks E, Sivachenko A, Cibulskis K, Kernytsky A, Garimella K, Altshuler D, Gabriel S, Daly M, et al: The Genome Analysis Toolkit: a MapReduce framework for analyzing next-generation DNA sequencing data. Genome Res 2010, 20(9):1297-1303.

14. Li H, Handsaker B, Wysoker A, Fennell T, Ruan J, Homer N, Marth G, Abecasis G, Durbin R, Genome Project Data Processing S: The Sequence Alignment/Map format and SAMtools. Bioinformatics 2009, 25(16):2078-2079.

15. Zhou $X 1$ RA: Prevention, diagnosis and treatment of high-throughput sequencing data pathologies. Molecular Ecology 2014, 23(7):1679-1700.

16. Criscuolo A, Brisse S: AlienTrimmer removes adapter oligonucleotides with high sensitivity in short-insert paired-end reads. Commentary on Turner (2014) Assessment of insert sizes and adapter content in FASTQ data from NexteraXT libraries. Front Genet 2014, 5:130.

17. Turner FS: Assessment of insert sizes and adapter content in fastq data from NexteraXT libraries. Front Genet 2014, 5:5.

18. Scythe. [https://github.com/vsbuffalo/scythe].

19. Baldi P, Brunak S, Chauvin Y, Andersen CA, Nielsen H: Assessing the accuracy of prediction algorithms for classification: an overview. Bioinformatics 2000, 16(5):412-424.

20. Langmead BaS SL: Fast gapped-read alignment with Bowtie 2. Nature Methods 2012, 9(4):357-359.
21. Guo W, Fiziev P, Yan W, Cokus S, Sun X, Zhang MQ, Chen PY, Pellegrini M: BS-Seeker2: a versatile aligning pipeline for bisulfite sequencing data. BMC Genomics 2013, 14:774.

22. Trapnell C, Pachter L, Salzberg SL: TopHat: discovering splice junctions with RNA-Seq. Bioinformatics 2009, 25(9):1105-1111.

23. Chen $X, X u H$, Yuan $P$, Fang F, Huss M, Vega VB: Integration of external signaling pathways with the core transcriptional network in embryonic stem cells. Cell 2008, 133(6):1106-1117.

24. Zhang Y, Liu T, Meyer CA, Eeckhoute J: Model-based analysis of ChIP-Seq (MACS). Genome Biology 2008, 9(9).

25. Henikoff JG, Belsky JA, Krassovsky K: Epigenome characterization at single base-pair resolution. PNAS 2011, 108(45):18318-18323.

26. Blake JA, Bult CJ, Eppig JT, Kadin JA: The Mouse Genome Database: integration of and access to knowledge about the laboratory mouse. Nucleic Acids Research 2014, , 42 Database: 810-817.

doi:10.1186/1471-2105-16-S1-S2

Cite this article as: Li et al:: PEAT: an intelligent and efficient paired-end sequencing adapter trimming algorithm. BMC Bioinformatics 2015 16(Suppl 1):S2.

\section{Submit your next manuscript to BioMed Central and take full advantage of:}

- Convenient online submission

- Thorough peer review

- No space constraints or color figure charges

- Immediate publication on acceptance

- Inclusion in PubMed, CAS, Scopus and Google Scholar

- Research which is freely available for redistribution

Submit your manuscript at www.biomedcentral.com/submit
C Biomed Central 